\title{
Comorbidity between obsessive-compulsive disorder and body dysmorphic disorder: prevalence, explanatory theories, and clinical characterization
}

\author{
This article was published in the following Dove Press journal: \\ Neuropsychiatric Disease and Treatment \\ 26 August 2015 \\ Number of times this article has been viewed
}

\author{
Álvaro Frías ${ }^{1,2}$ \\ Carol Palma ${ }^{1,2}$ \\ Núria Farriols 1,2 \\ Laura González ${ }^{2}$ \\ 'FPCEE Blanquerna, Universitat \\ Ramon Llull, Barcelona, ${ }^{2}$ Adult \\ Outpatient Mental Health Center, \\ Hospital de Mataró - CSdM, Mataró, \\ Spain
}

\begin{abstract}
Background: With the advent of the fifth edition of Diagnostic and Statistical Manual of Mental Disorders, body dysmorphic disorder (BDD) has been subsumed into the obsessive-compulsive disorders and related disorders (OCDRD) category.

Objective: We aimed to determine the empirical evidence regarding the potential relationship between BDD and obsessive-compulsive disorder (OCD) based on the prevalence data, etiopathogenic pathways, and clinical characterization of patients with both disorders.
\end{abstract}

Method: A comprehensive search of databases (PubMed and PsycINFO) was performed. Published manuscripts between 1985 and May 2015 were identified. Overall, 53 studies fulfilled inclusion criteria.

Results: Lifetime comorbidity rates of BDD-OCD are almost three times higher in samples with a primary diagnosis of BDD than those with primary OCD (27.5\% vs $10.4 \%)$. However, other mental disorders, such as social phobia or major mood depression, are more likely among both types of psychiatric samples. Empirical evidence regarding the etiopathogenic pathways for BDD-OCD comorbidity is still inconclusive, whether concerning common shared features or one disorder as a risk factor for the other. Specifically, current findings concerning third variables show more divergences than similarities when comparing both disorders. Preliminary data on the clinical characterization of the patients with BDD and OCD indicate that the deleterious clinical impact of BDD in OCD patients is greater than vice versa.

Conclusion: Despite the recent inclusion of BDD within the OCDRD, data from comparative studies between BDD and OCD need further evidence for supporting this nosological approach. To better define this issue, comparative studies between BDD, OCD, and social phobia should be carried out.

Keywords: obsessive-compulsive disorder, body dysmorphic disorder, comorbidity

\section{Introduction}

With the advent of the fifth edition of the Diagnostic and Statistical Manual of Mental Disorders (DSM-5) in 2013, body dysmorphic disorder (BDD) has been subsumed into the category of obsessive-compulsive disorders and related disorders (OCDRD) along with obsessive-compulsive disorder (OCD), hoarding disorder, trichotillomania, and excoriation disorder. Based on this conceptualization, BDD shares epidemiological, etiopathogenic, psychopathological, functional, evolutionary, and treatment-related features with OCD to a greater extent compared with empirical evidence from anxiety disorders or somatoform disorders. ${ }^{1}$ This assumption has been discussed over the last
Correspondence: Álvaro Frías Facultat de Psicologia, Ciencies de I'Educació i de l'Esport Blanquerna, C/Císter, 34, 08022 Barcelona, Spain Email afrias@csdm.cat 
20 years, resulting in an abundance of research from independent (BDD or OCD vs controls) and comparative studies (BDD vs OCD vs controls) so far.

Given this, several theoretical reviews have been previously carried out on this issue. ${ }^{2-5}$ Nonetheless, there are no theoretical reviews that include the latest findings on the comorbidity of BDD and OCD, neither epidemiological data, explanatory theories nor clinical characteristics. In addition, the previous reviews made inferences based on remarkable methodological divergences among the manuscripts gathered (eg, independent vs comparative studies). For this reason, we considered the need to carry out an up-to-date theoretical review with a more accurate methodological approach that would provide us with a better understanding of the potential relationship between OCD and BDD. Specifically, we sought to display main findings on three major issues, namely: 1) What is the prevalence of comorbidity between BDD and OCD? 2) Are there any etiopathogenic pathways that may account for the comorbidity between BDD and OCD? 3) What are the clinical characteristics of patients with comorbid OCD and BDD?

\section{Method}

\section{Search strategy}

A literature search was carried out in June 2015 through PsycINFO, Scopus, and PubMed databases from 1985 to
May 2015. Terms employed included indexing terms (eg, $\mathrm{MeSH}$ ) and free texts: ([body dysmorphic disorder OR BDD OR dysmorphophobia] AND [obsessive-compulsive disorder OR OCD] AND [comorbidity]).

\section{Selection criteria}

Inclusion criteria included studies in which: 1) some individuals were diagnosed with BDD and others with OCD or 2) some individuals were diagnosed with both disorders and were compared with others with one of these disorders alone. Psychiatric diagnosis should have been made according to DSM-III-R (1987) or DSM-IV (1994). Studies solely enrolling patients either with BDD or with OCD were excluded because they did not allow us to perform direct comparison between both disorders.

\section{Study selection}

Initial screening using search terms yielded 133 manuscripts (Figure 1). After reviewing all manuscripts, the authors agreed that 62 manuscripts available in English language could fulfill inclusion criteria and were potentially suitable for this review. Of them, four manuscripts were removed because they were not original findings (theoretical reviews) and five manuscripts were also ruled out because they were case reports with limited evidence, most of them comprising single case studies. Finally, 53 manuscripts fulfilled

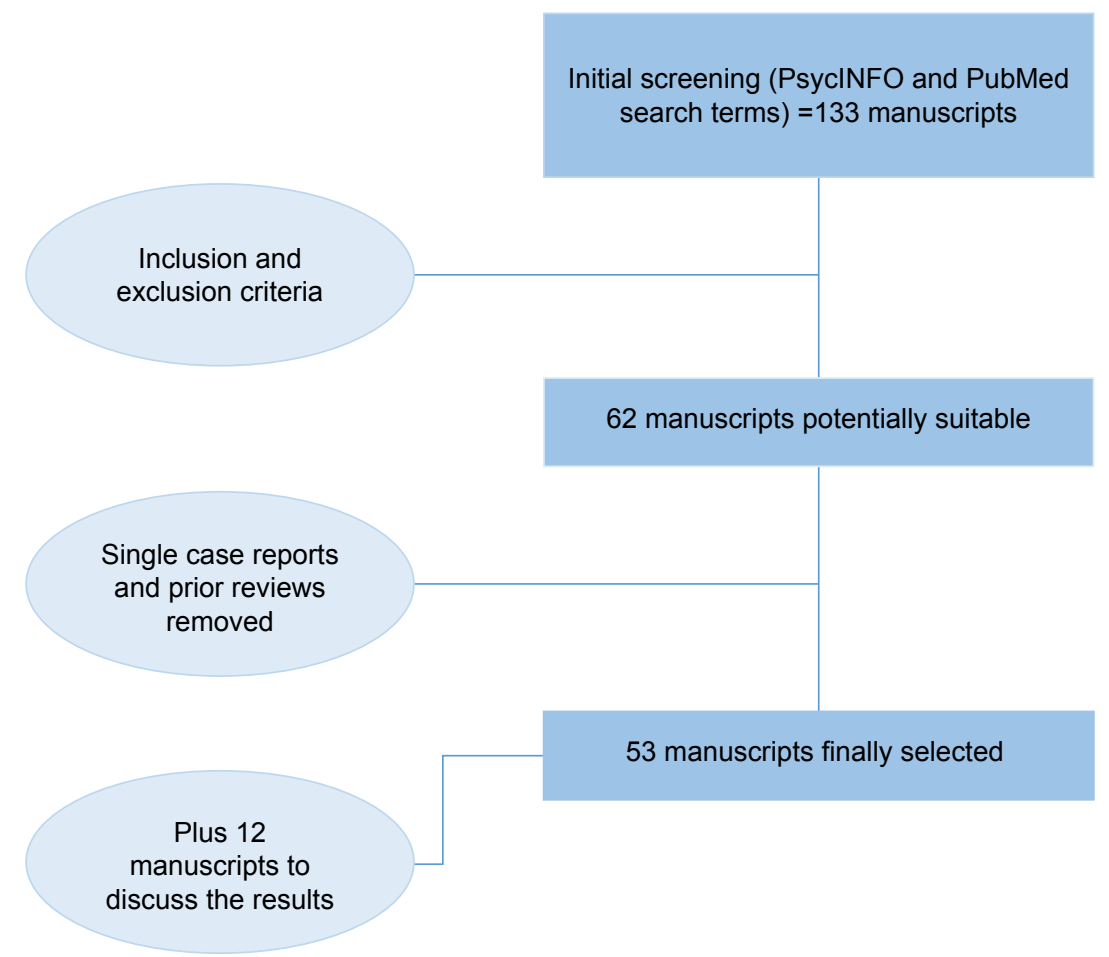

Figure I Flowchart of study selection. 
inclusion criteria and comprised this theoretical review. We added 12 related manuscripts to help with the discussion of the evidence.

\section{Data extraction}

Findings from each manuscript were distributed throughout the text, according to the following setup: 1) prevalence data; 2) etiopathogenic pathways; and 3) characteristics of patients with comorbid OCD and BDD. From a methodological approach, we also recorded for each manuscript: 1) sample size; 2) assessment instruments; 3) influence of confounding variables; 4) comparison group employed; and 5) outcome measures.

\section{Results}

\section{Prevalence of the comorbidity between $O C D$ and BDD}

The first goal of this review was to evaluate if patients with either OCD or BDD were likely to have the other diagnosis. For this purpose, we divided this paragraph into two sections: findings from samples with a primary diagnosis of OCD and those from samples with a primary diagnosis of BDD. Moreover, for each section, the results displayed were taken into account if they reflected lifetime or current comorbidity rates. Overall findings are illustrated in Figure 2.

\section{Prevalence of BDD in patients with a primary diagnosis of $O C D$}

Studies on the prevalence of BDD in individuals with a primary diagnosis of OCD have mostly enrolled adult outpatient samples from western OCD specialty centers. Concerning this issue, the lifetime prevalence of BDD within these samples ranged from $3 \%$ to $16 \%$ (mean $=10.4 \%)^{6-15}$ Specifically, data from the largest samples $(\mathrm{N}=901, \mathrm{~N}=457$, $\mathrm{N}=442$, and $\mathrm{N}=382$ ) yielded prevalence data from $8.7 \%$ to $15 \%$, relative to $3 \%$ in non-OCD patients. ${ }^{7,8,12,14}$ Conversely, the only study conducted in an Asian country (Indian sample, $\mathrm{N}=231$ ) consisting of both children and adults evidenced that $3 \%$ of them had comorbidity with BDD. ${ }^{9}$ The lower comparative prevalence could be the result of cross-cultural differences in BDD prevalence, although there is still no epidemiological data on the prevalence of primary BDD within Indian samples. Notwithstanding, a recent multicentric international study $(\mathrm{N}=457)$ found significant differences in comorbidity rates between subsamples (countries) with the highest prevalence evidenced in the Canadian OCD patients (up to $78 \%$ also met criteria for BDD, although statistical power was low because of the sample size). ${ }^{11}$ Regarding the current prevalence of BDD among OCD samples, studies are more limited and found that this type of comorbidity occurred in $3.8 \%-15.3 \%$ of OCD patients ( mean $=9 \%$ ) relative to $0 \%$ in non-OCD controls. ${ }^{16-18}$ Particularly, data from the study with the highest percentage should be taken with caution as: 1) BDD diagnosis was merely determined using a self-report screening instrument (Body Dysmorphic Disorder Questionnaire) and 2) the OCD sample was recruited from an inpatient psychiatric setting. ${ }^{18}$ Overall, neither the diagnosis criteria (DSM-III-R vs DSM-IV) nor the assessment instruments (the Structured Clinical Interview for DSM-IV [SCID-I] vs Mini International Neuropsychiatric Interview vs Schedule for Affective Disorders and Schizophrenia)

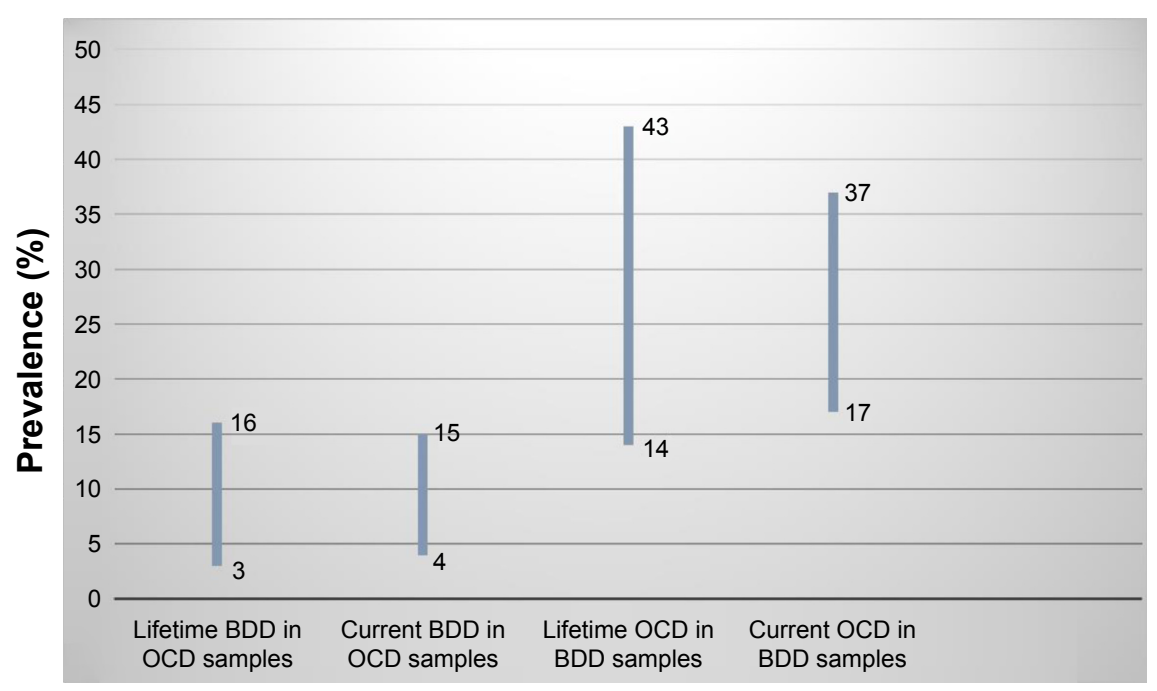

Figure 2 Percentage rates of comorbidity between OCD and BDD.

Note: Data presented as a $95 \%$ confidence interval.

Abbreviations: $O C D$, obsessive-compulsive disorder; BDD, body dysmorphic disorder. 
accounted for the differences in the prevalence data across the studies recruited.

\section{Prevalence of $O C D$ in patients with a primary diagnosis of BDD}

Researches on the prevalence of OCD in adult outpatients with a primary diagnosis of BDD have been scarce relative to those undertaken in OCD samples, albeit the methodology was more heterogeneous (eg, differences in recruitment and referral methods). Regarding this issue, the lifetime prevalence of OCD within BDD samples varied considerably from $43 \%$ to $14 \%$ (mean $=27.5 \%$ ). ${ }^{19-23}$ Particularly, data from the study indicated that the highest percentage should be considered with caution because the sample consisted of predominantly women subjects, referred to hospital centers for esthetical medical treatment (vs psychiatric setting). ${ }^{19}$ In addition, the study with the lowest percentage was initially composed of a drug-free sample with a primary diagnosis of major depression in which further comorbidity assessments were conducted. ${ }^{22}$ Concerning the current prevalence of OCD among BDD samples, rates for this type of comorbidity were $37 \%-16.7 \%$ in $\mathrm{BDD}$ patients (mean $=25.7 \%$ ) relative to $8 \%$ in non-BDD patients. ${ }^{21,24-26}$ Specifically, data from the study with the largest sample $(\mathrm{N}=293)$ indicated that $25 \%$ of those with BDD also had OCD. ${ }^{21}$ Similar to OCD samples, neither the diagnostic criteria nor the assessment instruments explained the differences in the rates of comorbidity across the studies.

\section{Is there any etiopathogenic pathway that may account for the comorbidity of BDD and OCD?}

The second goal of this review was to assess potential etiopathogenic pathways that might explain the comorbidity of
BDD and OCD. The main findings concerning: 1) common shared factors (genetics and environmental influences, sociodemographic profile, cognitive correlates, personality features, psychiatric comorbidity, and symptom co-occurrence); 2) one disorder being a risk factor for the other (vulnerability model); and 3) chance association (random co-occurrence) are described. The overall results from the common shared factors are illustrated in Figure 3.

\section{Common shared factors (third variables)}

\section{Genetics and environmental influences}

Studies assessing rates of lifetime psychiatric diagnosis in the first-degree relatives of patients with either OCD or BDD reveal greater percentage of both disorders among their familiars, compared with the first-degree relatives of healthy subjects ${ }^{19}$ or non-OCD patients. ${ }^{6,7}$ In addition, these studies showed higher prevalence for mental disorders other than $\mathrm{OCD} / \mathrm{BDD}$ among the first-degree relatives of $\mathrm{OCD} /$ BDD patients, namely, eating disorders, ${ }^{19}$ social phobia, ${ }^{7,16}$ and major depression. ${ }^{7,13,16}$ Except for the most recent study, ${ }^{7}$ these findings should be taken cautiously because of a low statistical power ${ }^{6}$ or the absence of direct assessment of firstdegree relatives by using the Family History Screen ${ }^{16,19}$ or telephone procedures. ${ }^{13}$

Overall, findings on psychiatric family history of patients with OCD, BDD, or both have been employed to suggest a particular link between them, specifically regarding the inclusion of both disorders into an OCDRD taxonomy. ${ }^{27}$ Accordingly, further studies have been carried out to investigate whether common genetics or environmental factors could account for this potential relationship. For instance, a study within a community sample of 2,148 adult twins revealed that the covariation between BDD and OCD traits was largely accounted for by genetic influences common (64\%) to both

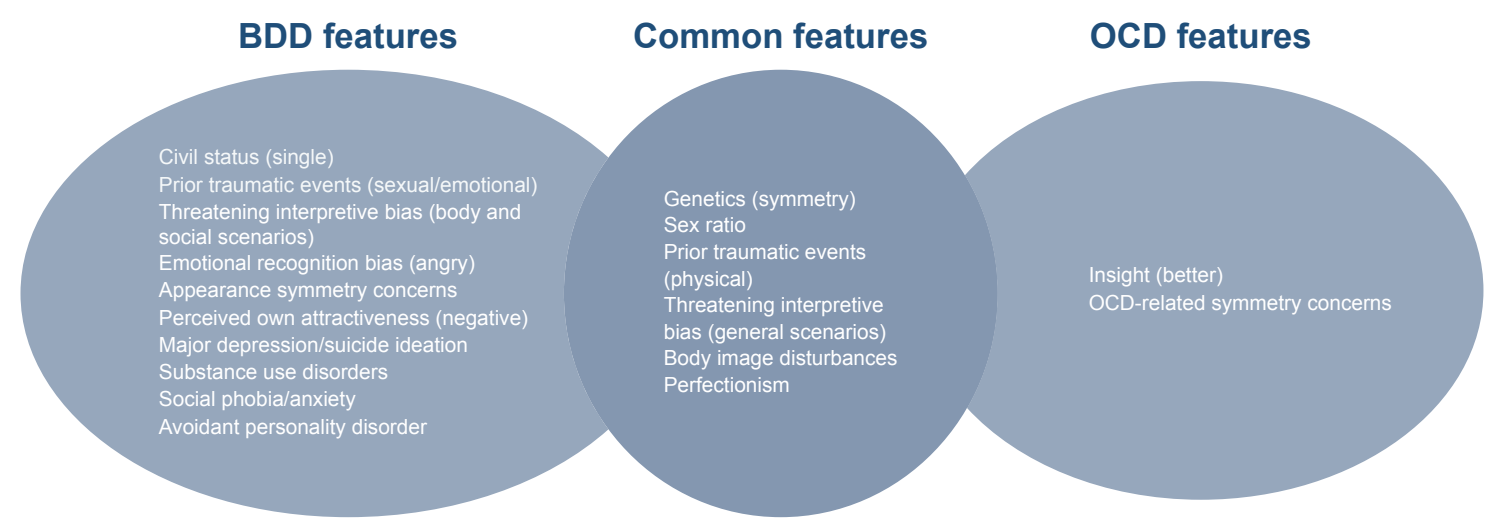

Figure 3 Divergences and similarities between patients with OCD vs BDD from comparative studies. Abbreviations: $O C D$, obsessive-compulsive disorder; BDD, body dysmorphic disorder. 
phenotypes. ${ }^{28}$ This genetic overlap was even higher when specific OC symptom dimensions were considered; up to $82 \%$ of the phenotypic correlation between the obsession and symmetry-ordering symptom dimensions and BD concerns were attributable to common genetic factors. Despite these findings, the limitations of this study should be taken into account, specifically the exclusion of male twins and the use of self-report measures (Dysmorphic Concern Questionnaire) instead of structured clinical interviews that could increase the risk of a false-positive diagnosis. Later, the same group of research performed a latent class analysis among 5,408 female twins (3,041 monozygotics and 2,367 dizygotics) assessed for OCDRD. ${ }^{29}$ The authors found that common (vs disorder-specific) genetic influences were moderate (33\%) in BDD when all the OCDRD (including OCD) were analyzed together. In addition, shared (vs non-shared) environmental risk factors in BDD were low $(10 \%)$ when all the OCDRD were considered. Concerning the presence of common environmental risk factors, an additional crosssectional study found that BDD patients reported higher rates of sexual ( $28 \%$ vs $2 \%$ ) and emotional abuse ( $22 \%$ vs $6 \%$ ), but similar rates of physical abuse (14\% vs $8 \%$ ) than OCD patients. ${ }^{30}$ However, methodological flaws, such as potential recall/misinterpretation biases and the lack of standardized instruments to assess traumatic events, may have hindered the validity of these findings.

\section{Sociodemographic profile}

There is consistent evidence regarding similar sex ratios in BDD and OCD. ${ }^{13,30-35}$ The only study that revealed significantly higher rates of BDD than OCD (65\% vs $41 \%)$ in women arose from the smallest sample of all the studies collected (BDD, $\mathrm{N}=23$ vs $\mathrm{OCD}, \mathrm{N}=22$ ). With respect to marital status, the findings are heterogeneous after controlling for age, indicating either similar marital status for both disorders $\mathrm{s}^{30,31,33,34}$ or greater rates of BDD among unmarried/ single patients. ${ }^{13,32}$ Methodologically noticeable, the former studies included patients with lifetime diagnoses of OCD or BDD, while the latter studies recruited patients with current diagnosis of BDD or OCD. Overall, several of these studies have not addressed whether between-group differences in the current symptom severity accounted for the findings in marital status. ${ }^{30,32,34}$

\section{Psychopathological features}

BDD patients often spend hours each day performing ritualistic behaviors, such as excessive grooming, mirror checking, repetitive touching, excessive application of makeup, and camouflaging one's appearance with clothing or jewelry. ${ }^{13}$ These repetitive behaviors have been postulated to be similar to compulsions performed by OCD patients. Regardless of this theoretical assumption, empirical research on the potential common psychopathological features for both disorders has been focused on the degree of insight regarding OCD and BDD. Concerning this issue, cross-sectional studies have found poorer insight for BDD than for OCD in patients with comorbid conditions or in those with the former diagnosis when compared with subjects with the latter diagnosis. These findings have been consistent, irrespective of the assessment instrument used, that is, single-items from the Yale-Brown Obsessive Compulsive Scale (Y-BOCS), ${ }^{14}$ the multidimensional approach from the Brown Assessment of Beliefs Scale (BABS), ${ }^{33,36-38}$ or the Overvalued Ideas Scale. ${ }^{39}$

\section{Cognitive correlates}

It has been suggested that one cognitive correlate that predisposes or maintains both BDD and OCD could be a threatening interpretive bias toward ambiguous stimuli either general or disorder specific. Regarding this theme, there is only one experimental study in which the patients chose one alternative of three possible options that attempted to follow an unfinished narrative story concerning several issues. The authors found that OCD and BDD patients exhibited a threatening interpretive bias for general scenarios relative to healthy controls, but only those with BDD reported a threatening interpretive bias for body-related scenarios and for social scenarios..$^{40}$ The sample size was quite small (BDD, $\mathrm{N}=19$ and $\mathrm{OCD}, \mathrm{N}=20$ ), and there were no replication studies. Thus, this finding should be taken cautiously. In addition, other studies by the same group of researchers have been conducted to determine whether both disorders are characterized by an emotional recognition bias. With respect to this issue, one study using the facial photographs from the Ekman and Friesen' $\mathrm{s}^{41}$ set evidenced that BDD individuals, relative to OCD and healthy controls, were less accurate in identifying facial expressions of emotion and more often misidentified emotional expressions as angry. This study was also carried out with a small sample (BDD, $N=20$ and $O C D, N=20$ ) and warrants further replication.

Other studies have addressed whether OCD/BDD patients could manifest cognitive reasoning biases that were either general or disorder specific. Regarding this issue, 20 OCD, $20 \mathrm{BDD}$, and 20 healthy controls completed a memory task assessing reality-monitoring ability for verbal stimuli (neutral, negative, BDD-related, and OCD-related stimuli), that is, the ability to discriminate between imagination and reality. 
There were no significant differences between the three groups for each type of stimuli in spite of a lower degree of insight in the BDD group as measured by the BABS. ${ }^{38}$ Although this research has its limitations, further studies with larger samples and more ecological stimuli (images rather than words) should clarify the potential role of this cognitive ability in both disorders. In addition, this group research also assessed whether the same sample could reveal betweengroup differences in jump-to-conclusions reasoning bias, that is, the need to request significantly less information before making a decision. Accordingly, all participants completed two tests of probabilistic reasoning: the beads task (neutral task) and the survey task (self-relevant task). Similarly, there were no significant between-group differences, despite lower level of insight in BDD patients. ${ }^{42}$

Other studies have been focused on measuring esthetic thoughts in both disorders. First, one research study showed non-personal photos displaying faces varying in attractiveness (attractive, average, and unattractive) and then other facial photos taken of the study participants. The individuals were asked to rate the photos from both phases in terms of their physical attractiveness. Similar to OCD and healthy controls, BDD patients endorsed normative ratings of beauty (standards of attractiveness), but their own perceived attractiveness was significantly lower compared with the other groups. ${ }^{43}$ This study was also conducted with a small sample (BDD, N=19 and OCD, N=21) and needs further replication. In addition, one previous study with a large population comprising, among others, 100 patients with OCD and 100 individuals with BDD found that $20 \%$ of the BDD patients had an occupation or education in art or design compared with $3 \%$ of those with OCD. ${ }^{35}$ The authors underlined that this finding could reflect a higher esthetic sensitivity among BDD. Notwithstanding, this interpretation should be taken cautiously because of the cross-sectional nature of this study and the use of questionnaires rather than structured clinical interviews to make the psychiatric diagnosis.

Other types of studies have focused on symmetry. An experimental study using non-personal photos varying in facial symmetry showed that 20 BDD patients reported similar perceptual ability and evaluative preference for symmetry as 20 OCD patients and 20 healthy controls. ${ }^{44}$ Notwithstanding, data from similar studies with larger samples, which may incorporate manipulated patients' faces, should be considered to determine if BDD sufferers could apply different standards to their own face than they do to others' faces. Concerning this proposed methodology, a pilot study that used scanned photography of OCD patients, BDD patients, and healthy controls found that both clinical patients modified over $50 \%$ (vs $0 \%$ in controls) of undistorted photographs because they thought that these stimuli did not exactly reflect as they looked like. ${ }^{45}$ The authors suggested that this finding indicated a similar body image disturbance in OCD and BDD, although statistical power was poor because of a very small sample (OCD, N=10 and BDD, N=10). Furthermore, another study with a large sample of 275 BDD patients found than $31 \%$ of participants reported appearance-related symmetry concerns for at least one body part with which they were preoccupied. In addition, this subset of BDD patients was more likely to have lifetime OCD in general (48\% vs $25.5 \%$ ), but not more lifetime OCD-related symmetry concerns specifically (36\% vs 38\%), suggesting different mechanisms in BDD and OCD symmetry concerns. ${ }^{46}$ Notwithstanding, appearance-related symmetry concerns were measured by a single-item from the BDD data form. Thus, these findings could be limited because of the assessment instrument.

\section{Personality features}

Personality characteristics have been rarely assessed and have arisen from divergent perspectives (categorical vs dimensional approaches). On the one hand, an American study using a mixed sample (inpatients and outpatients) diagnosed with OCD $(\mathrm{N}=210)$ and BDD $(\mathrm{N}=45)$ found no significant between-group differences in the prevalence of personality disorders as ascertained by SCID-II. ${ }^{33}$ The authors only evidenced higher rates (trend level) of obsessive-compulsive personality disorder in OCD than in BDD individuals $(26 \%$ vs 9\%). Regarding OC traits, a study with a small sample (OCD, $\mathrm{N}=21$ and $\mathrm{BDD}, \mathrm{N}=19$ ) did not reveal differences in perfectionism between OCD and BDD patients as measured by the Multidimensional Perfectionism Scale. However, both clinical groups reported more perfectionism than the healthy controls. On the other hand, an additional Turkish study with a moderate-sized sample, drug free before the recruitment, showed that BDD patients $(\mathrm{N}=29)$ were more likely than OCD patients $(\mathrm{N}=49)$ to have narcissistic $(31 \%$ vs $6 \%$ ), histrionic ( $38 \%$ vs $12 \%$ ), and avoidant (66\% vs $33 \%$ ) personality disorders as measured by SCID-II. ${ }^{34}$

Psychiatric comorbidity and symptom co-occurrence Comparative rates of comorbidity in OCD and BDD have mainly focused on depressed mood (either dimensional or categorical approach), social anxiety (either dimensional or categorical approach), eating disorders, and drug misuse. Regarding depressed mood, studies have yielded mixed results. Several studies found similar rates of lifetime major 
depressive disorder (MDD) for both clinical groups, up to $53 \%$ for each group, ${ }^{32,34}$ while others showed that BDD patients were more likely to have MDD relative to OCD individuals. ${ }^{13,33}$ Particularly, the study with the largest sample (OCD, $\mathrm{N}=210$ and BDD, $\mathrm{N}=45$ ) found significant differences of MDD comorbidity when comparing both clinical groups with a peak of $87 \%$ in BDD and $65 \%$ in OCD. ${ }^{33}$ It should be underscored that, unlike the studies yielding between-group differences, those who found similar rates were conducted in non-American samples. Concerning the severity of current depressive mood as measured by questionnaires, the studies tend to reveal similar severity for OCD and BDD. $33,34,39,45$ The only study showing greater severity of depressive mood on BDD had a small sample (BDD, N=19 and OCD, $\mathrm{N}=20$ ) and lacked statistical power. ${ }^{40}$ In addition, relative to OCD patients, those with BDD have reported greater rates of lifetime suicidal ideation in general (78\% vs 55\%) because of their disorder specifically ( $22 \%$ vs $8 \%),{ }^{13,33}$ but their current suicidal ideation is similar to OCD individuals ( $27 \%$ vs $20 \%)^{32}$

Concerning lifetime social phobia, most of the study showed similar rates of comorbidity in OCD and BDD patients, up to $27 \%$ and $31 \%$, respectively. ${ }^{32-34}$ There are research results yielding significant higher rates of lifetime social phobia in BDD than in OCD (49\% vs 19\%). Notwithstanding, when assessing severity of social anxiety, BDD individuals reported significant higher scores on the Fear of Negative Evaluation scale than OCD patients. ${ }^{40}$ In spite of this fact, the sample size of this study was small (OCD, N=20 and $\mathrm{BDD}, \mathrm{N}=19$ ) and the findings need further replication.

With regard to lifetime eating disorders, the studies within American samples found similar rates among patients with a primary diagnosis of either OCD or BDD, with percentages up to $13 \%$ and $7 \%$, respectively. ${ }^{13,33}$ Conversely, an Italian study found significantly higher rates of eating disorders in BDD relative to OCD patients with percentages up to $18 \%$ and $9 \%$, respectively. ${ }^{32}$

Finally, rates of comorbid lifetime alcohol-substance use disorders (SUD) have been more usually found in BDD than in OCD with the quantity of primary diagnoses up to $47 \%$ and $24 \%$, respectively. ${ }^{32,33}$ The only study that showed nonsignificant between-group differences in alcohol-SUD used the smallest sample (OCD, $\mathrm{N}=53$ and $\mathrm{BDD}, \mathrm{N}=53) .{ }^{13}$

\section{One disorder being a risk factor for the other}

One indirect approach to take to discover whether one disorder may be a risk factor for the other may be by retrospectively determining the age at the onset of both disorders, either in patients with comorbid OCD and BDD or in comparative studies of OCD vs BDD. Accordingly, if one disorder tends to appear substantially before the onset of the other, this could provide some evidence to this issue.

Regarding this theme, qualitative data from these types of studies indicate that BDD usually precedes $\mathrm{OCD},{ }^{21}$ but, except for one research study, ${ }^{32}$ quantitative data reveal that the mean age of onset for both disorders does not significantly differ $(\mathrm{OCD}=17.8$ vs $\mathrm{BDD}=17.6){ }^{13,14,17,30,33,39}$

\section{Chance association}

Another heuristic approach to explain the comorbidity of OCD and BDD could be that the co-occurrence of both disorders was a clinical artifact linked to psychiatric samples. That is, the likelihood that one disorder was associated with the other would be higher in psychiatric samples than in the general population because individuals from the psychiatric samples would be more psychopathologically ill (Berkson's bias). If so, patients within these samples should also have greater comorbidity with other mental disorders than OCD or BDD specifically.

Accordingly, several studies have addressed whether patients with a primary diagnosis of either OCD or BDD present high rates of comorbid mental disorders other than OCD or BDD. Regarding this issue, research in BDD samples has systematically showed greater rates of lifetime and current social phobia than OCD, ${ }^{12,15,17,22,26}$ with the exception of one study enrolling female cosmetic surgery patients. ${ }^{19}$ Specifically, the study with the largest BDD sample $(\mathrm{N}=293)$ found greater rates of social phobia and MDD than OCD. ${ }^{21}$ The same finding was evidenced in other study carried out with a smaller BDD sample $(\mathrm{N}=54) .{ }^{25}$ Concerning the studies in OCD samples, research on psychiatric comorbidity beyond BDD has been scarce. First, one study showed that current comorbidity rates of social phobia, generalized anxiety disorder, and panic disorder were higher than BDD among obsessive patients. ${ }^{16}$ In addition, two studies with larger OCD samples ( $\mathrm{N}=901$ and $\mathrm{N}=457$ ) reported greater lifetime comorbidity rates of social phobia and MDD than with BDD among obsessive patients. ${ }^{8,11}$

\section{Disentangling the core features of patients with comorbid BDD and OCD}

The third goal of this review was to describe the core features of patients with comorbid BDD and OCD to determine both the clinical impact of one disorder on the other (eg, insight, content, severity, age at onset, and response to treatment) 
and the distinctive clinical characteristics of subjects with comorbid OCD and BDD relative to those with one diagnosis alone (eg, sociodemographic characteristics, comorbidity rates, and quality of life).

\section{Findings from comparative studies between comorbid OCD-BDD and OCD patients}

\section{Impact of BDD on OCD characteristics}

With regard to OCD insight, comparative studies between patients with comorbid OCD-BDD and OCD patients have found either similar ${ }^{14,47}$ or poorer degree of insight among those with comorbid BDD. ${ }^{8,48}$ Most of these studies have assessed the insight using the BABS with the exception of one study with a poorer assessment instrument that used a single-item from the Y-BOCS. ${ }^{14}$ Among those evidencing between-group differences, researchers have also sought to control for confounding variables (eg, severity of OCD). Concerning this issue, one study with a large sample ( $\mathrm{N}=901)$ showed that comorbid BDD was not associated with poorer insight into $\mathrm{OCD},{ }^{8}$ while another smaller sample $(\mathrm{N}=103)$ confirmed this relationship. ${ }^{48}$ Concerning OCD severity as measured by the Y-BOCS, researchers usually have found no between-group differences. ${ }^{8,14,18,34,47,48}$ Regarding OCD symptomatology as assessed by the Y-BOCS, comparative research has yielded mixed results - either no between-group differences ${ }^{47}$ or greater severity or presence of OC symptoms in those with comorbid BDD, such as somatic obsessions, ${ }^{48}$ sexual obsessions, ${ }^{8,14}$ religious obsessions,${ }^{8}$ aggressive obsessions, ${ }^{8}$ symmetry $\mathrm{O} / \mathrm{C},{ }^{18}$ hoarding $\mathrm{O} / \mathrm{C},{ }^{18,48}$ or checking compulsions. ${ }^{14,18}$ In spite of these facts, only one study with 103 OCD patients showed that some OC contents (somatic and hoarding obsessions) were associated with the presence of comorbid BDD after performing a regression analysis. ${ }^{48}$ Concerning age of onset of $\mathrm{OCD}$, comparative studies agree; there is earlier onset among those with comorbid BDD. ${ }^{8,18,48}$ Finally, both randomized, controlled trials with antidepressants ${ }^{47,49}$ and naturalistic treatments with psychopharmacology and psychotherapy ${ }^{14,18}$ showed that treatment response for OCD was independent of the presence of comorbid BDD.

\section{Distinctive, non-OCD characteristics of comorbid BDD-OCD patients}

Concerning sex ratio, comparative studies show similar sex distribution between BDD-OCD and OCD patients. ${ }^{13,14,31-34,47}$ Regarding civil status, studies find higher rates of single people among BDD-OCD patients (vs OCD) ${ }^{8,13,18,32}$ than no between-group differences, ${ }^{31,34}$ even after controlling for age. ${ }^{32}$ Rates of employment and education level are consistently poorer in OCD-BDD patients relative to OCD patients. ${ }^{8,13,32,33,48}$ Concerning psychiatric comorbidities, patients with BDD-OCD showed similar rates of lifetime MDD than those with OCD..$^{8,13,33,34,47}$ However, they also reported higher current depressive symptom severity as well

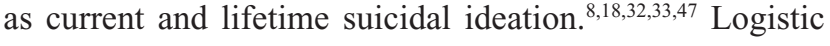
regression analysis also confirmed the association between the latter two variables and comorbid BDD. ${ }^{8,33}$ Moreover, both lifetime social phobia and alcohol-SUD have consistently been more likely in OCD-BDD patients than in OCD individuals. ${ }^{18,32-34,47,48}$ Particularly, the association between BDD comorbidity and the presence of social phobia was confirmed in OCD samples using logistic regression analysis. ${ }^{8}$ Lifetime rates of eating disorders have yielded mixed results, either no between-group differences in American samples ${ }^{13,33}$ or higher prevalence in BDD-OCD patients compared to OCD patients among non-American samples. ${ }^{8,32}$ Specifically, using logistic regression analysis, one study with a large OCD sample ( $\mathrm{N}=901)$ revealed that BDD comorbidity was associated with the presence of eating disorders among OCD patients. ${ }^{8}$ With regard to the assessment of personality disorders, the research is scarce, but tends to find greater rates of either Cluster B personality disorders or avoidant personality disorders in OCD patients with (vs without) comorbid BDD, even after controlling for OCD severity. ${ }^{33,34}$ Finally, as suggested by the previous results, a single study $(\mathrm{N}=250)$ found that the presence of BDD comorbidity was associated with a poorer quality of life among those with a primary diagnosis of OCD, even after controlling for OCD severity ${ }^{31}$ This study used several rigorous psychometric instruments such as the Longitudinal Interval Follow-Up Evaluation.

\section{Findings from comparative studies between comorbid OCD-BDD and BDD patients Impact of OCD on BDD characteristics}

Research on the impact of OCD on BDD is scarce and inconclusive. With regard to the insight into BDD, one single study found lower insight in patients with comorbid OCD-BDD than in BDD patients. ${ }^{50}$ However, this study used limited methodology, that is, insight was measured by a single-item from the Y-BOCS. The statistical power was low due to the sample size $(\mathrm{N}=30)$, and the sample was predominantly men (83\%). Concerning the severity of BDD as measured by the BDD-YBOCS, one study showed greater symptom severity in comorbid patients, ${ }^{33}$ while another did not find between-group differences. ${ }^{50}$ The former study had a more adequate sample 
size $(\mathrm{N}=85$ vs $\mathrm{N}=30)$ and was methodologically more relevant. Concerning the content of BDD as ascertained by the BDDYBOCS, there is only one study with a moderate sample size $(\mathrm{N}=58)$, which evidenced greater face concerns among those with BDD alone (vs BDD-OCD patients). Comparative studies focused on the age of onset of BDD have not found betweengroup differences. ${ }^{34,50}$ Finally, both clinical trials with antidepressants and naturalistic treatments with psychopharmacology and psychotherapy showed that the presence of comorbid OCD was associated with a similar treatment response for BDD, even after controlling for confounding variables. ${ }^{24,51}$

\section{Distinctive, non-BDD characteristics of BDD-OCD patients}

Research on this issue is scarce and lacks methodological rigor because of the moderate sample size and the absence of secondary analysis to control for confounding variables with the exception of one study. ${ }^{33}$ Regarding sociodemographic characteristics, research has consistently found similar sex ratios, numbers of married people, employment rates, and education levels in OCD-BDD and BDD patients, ${ }^{13,31,33,34,50}$ even after controlling for age. ${ }^{32}$ Concerning psychiatric comorbidities, studies on lifetime MDD have yielded mixed results, ${ }^{33,34}$ finding even lower rates of MDD among BDDOCD patients when compared with BDD individuals. ${ }^{13}$ In addition, the presence of OCD comorbidity was not associated with current depressive symptom severity as measured by questionnaires among patients with a primary diagnosis of BDD. ${ }^{33,34}$ Concerning lifetime and current suicidal ideation, OCD-BDD patients were more likely to have suicidal thoughts than those with BDD alone, ${ }^{13,32}$ but differences disappeared when controlling for BDD severity. ${ }^{33}$ Despite methodological similarities between the studies, lifetime comorbidity of social phobia, eating disorders, and alcoholSUD have yielded mixed results, either higher prevalence in BDD-OCD patients ${ }^{32}$ or similar rates in BDD-OCD and BDD patients. ${ }^{13,33,34}$ Likewise, researches on personality disorders have also found similar rates in BDD-OCD and BDD patients. ${ }^{33,34}$ Finally, a single study $(\mathrm{N}=250)$ found that the presence of OCD comorbidity was not associated with a poorer quality of life among those with a primary diagnosis of BDD, after controlling for BDD severity. ${ }^{31}$

\section{Discussion}

\section{Prevalence of the comorbidity between $O C D$ and $B D D$}

Findings from this review underscore that the rates of BDD OCD comorbidity ranges from $3 \%$ to $43 \%$ and are almost threefold higher in BDD samples than in OCD samples. How can we explain these divergences between both types of samples? We postulate that only some specific OCD symptoms, mainly symmetry obsessions, could have a relationship with BDD. This suggestion is partly supported by genetic studies ${ }^{28}$ but still has not received evidence from comparative studies (BDD-OCD vs OCD) on OCD symptomatology. Hence, further studies should investigate this issue. Furthermore, prevalence studies tend not to include a control group (healthy or non-OCD/BDD controls) when assessing rates of comorbidity of both disorders. ${ }^{67,17,26}$ Thus, despite high rates of this comorbidity, we cannot establish an odds ratio, and this limitation should be taken into account by future research. Besides, the studies collected arise from psychiatric samples, mainly OCD specialty clinics. Hence, the comorbidity rates may be inflated due to recruitment bias and cannot be generalized to the general population. Further research on community samples could help to obtain more accurate and firm evidence on the comorbidity rates. Likewise, clinicians should take into account that high rates of comorbidity of BDD and OCD do not necessary imply a distinctive relationship between both disorders. Indeed, prevalence studies have even found greater comorbidity with social phobia and MDD than with OCD and BDD in patients with one of the two latter diagnoses.

\section{Is there an etiopathogenic pathway that may account for the comorbidity of BDD and OCD?}

Findings from this review stress that there is still no sound evidence concerning potential etiopathogenic pathways that may explain comorbid OCD and BDD. Although methodologically flawed, retrospective studies consistently report similar age of onset for both disorders. This finding partly rules out the likelihood that one disorder was a risk factor for the other (vulnerability model), although prospective studies should be undertaken to clarify this theme. Concerning the chance association approach, findings from this review underscore that both OCD and BDD samples present high comorbid rates with other mental disorders than with each other. Nevertheless, this does not necessarily imply that there was random co-occurrence of OCD and BDD. Rather, this may suggest that BDD may represent a heterogeneous nosological category without a univocal and complete relationship with OCD. Regarding the presence of common shared factors (third variables), more divergences than similarities have been found when both disorders are directly compared. BDD patients tend to manifest a more 
severe clinical profile, both psychopathological and functional, when compared with OCD individuals. Additionally, many promising fields of research (eg, cognitive correlates) have not been adequately investigated and lack enough study subjects to provide compelling evidence. Moreover, other external criteria have been rarely addressed, despite yielding similar findings for OCD and BDD in independent studies. For instance, comparative studies on neurobiological (findings from limbic, visual cortex, and frontostriatal cortex), psychopharmacological, and neuropsychological correlates are almost nonexisten ${ }^{45,52-55}$ and should be investigated by future research. Maybe, the most consistent and intriguing finding observed in comparative studies has been the low degree of insight into their disorder among BDD patients, most of whom evidence poor or lack of insight. This finding raises a clinical doubt whether appearance-related concerns in BDD patients should be considered an intrusive thought or, otherwise, an overvalued idea or a delusional thought. We thought that some indirect clues may be gathered from other comparative studies collected to partly elucidate this issue. For instance, cognitive reasoning biases traditionally observed in delusional patients (eg, jumping to conclusions and reality-monitoring deficits) have not been found in BDD individuals. In addition, there is preliminary evidence suggesting a high esthetic sensitivity in BDD patients, ${ }^{35}$ which may indicate that appearance-related concerns may be egosyntonic and not a random thought. Taking into account all these findings, we suggest that appearance-related concerns in BDD patients are more closely related to overvalued ideas than to intrusive or delusional thoughts as defined by phenomenological authors. ${ }^{56,57}$ If so, motivational interviewing as well as inference-based therapy may be a key component in the treatment of BDD (vs OCD) patients who are not willing to change this psychopathology because of their egosyntonic features. ${ }^{58,59}$

Despite the fact that this review has not completely followed PRISMA guidelines,${ }^{60}$ which are currently considered the gold standard in the field, we consider that the recent integration of BDD into OCDRD needs further confirmation. Hence, we underscore that the recent inclusion of BDD within OCDRD (as classified in the DSM-5 in 2013 and proposed in the International Classification of Diseases 11th Revision [ICD-11 $]^{61}$ ) should be taken cautiously. Alternatively, the inclusion of BDD as a type of hypochondriacal disorder (ICD-10) also seems not to collect enough evidence from empirical research. ${ }^{62}$ In fact, data from these comparative studies almost provide more indirect evidence on the potential similarities between BDD and social phobia (eg, threatening interpretive bias for social scenarios, emotional recognition bias for angry expressions, comorbidity rates of social phobia and avoidant personality disorder, family loading, and so on). This assumption is in line with the Asian approach to BDD as a possible social phobia subtype (in Japanese: taijin kyofusho [fear disorder of interpersonal relationships]). ${ }^{63}$ As a consequence, there is a compelling need to carry out comparative studies between OCD, BDD, and social phobia to redefine this issue. Maybe, by performing these types of studies, we can pose whether there might be two BDD profiles: a reassurance/compulsive subtype of BDD as defined in DSM- $5^{64}$ (with repetitive behaviors or mental acts), closely related to OCD, and a phobic (avoidant) subtype of BDD, closely related to social phobia.

\section{Disentangling the core features of patients with comorbid BDD and OCD}

Findings from this review stress that the clinical impact of one disorder (OCD or BDD) on the other is the exception rather than the rule. Notwithstanding, there is a need to undertake more comparative studies between comorbid OCD-BDD and $\mathrm{BDD}$ to rule out the previous assumptions. Of interest, clinicians should suspect the potential of an overlooked or hidden BDD comorbidity diagnosis when age of OCD onset is earlier than expected. Unlike OCD comorbidity in patients with a primary diagnosis of $\mathrm{BDD}$, there is also consistent evidence indicating that the presence of BDD comorbidity exerts a cumulative negative impact on OCD individuals, both functional (eg, poorer quality of life, more unemployment, and living alone) and psychopathological (more comorbidity with social phobia, alcohol-SUD, Cluster B personality disorders, and suicidal ideation). Hence, clinicians usually treating OCD patients in specialty clinics should take special care of those with comorbid BDD because of their greater clinical severity and psychosocial impairment, which in turn make them more difficult to attend to in clinical settings as therapeutic adherence can be hampered by their clinical severity and dysfunctionality. Concerning this issue, an additional unexplored field of research deals with the combined treatment of OCD and BDD in those being diagnosed with this comorbidity. ${ }^{23}$ This is particularly striking as a 3-year prospective study with a small sample of OCD-BDD patients preliminarily found that improvements in OCD predicted BDD remission (but not vice versa) ${ }^{65}$ Hence, further research aimed at treating comorbid BDD is mandatory among OCD patients.

\section{Disclosure}

The authors report no conflicts of interest in this work. 


\section{References}

1. Hollander E. Obsessive-compulsive disorder and spectrum across the life span. Int J Psychiatry Clin Pract. 2005;9(2):79-86.

2. Chosak A, Marques L, Greenberg JL, Jenike E, Dougherty DD, Wilhelm S. Body dysmorphic disorder and obsessive-compulsive disorder: similarities, differences and the classification debate. Expert Rev Neurother. 2008;8(8):1209-1218.

3. Díaz Mársá M, Carrasco JL, Hollander E. Body dysmorphic disorder as an obsessive-compulsive spectrum disorder. Actas Luso Esp Neurol Psiquiatr Cienc Afines. 1996;24(6):331-337.

4. Phillips KA, McElroy SL, Hudson JI, Pope HG Jr. Body dysmorphic disorder: an obsessive compulsive spectrum disorder, a form of affective spectrum disorder, or both? J Clin Psychiatry. 1995;56(4): 41-51.

5. Phillips KA, Kaye WH. The relationship of body dysmorphic disorder and eating disorders to obsessive-compulsive disorder. CNS Spectr. 2007; 12(5):347-358.

6. Bienvenu OJ, Samuels JF, Riddle MA, et al. The relationship of obsessive-compulsive disorder to possible spectrum disorders: results from a family study. Biol Psychiatry. 2000;48(4):287-293.

7. Bienvenu OJ, Samuels JF, Wuyek LA, et al. Is obsessive-compulsive disorder an anxiety disorder, and what, if any, are spectrum conditions? A family study perspective. Psychol Med. 2012;42(1):1-13.

8. Conceição Costa DL, Chagas Assunção M, Arzeno Ferrão Y, et al. Body dysmorphic disorder in patients with obsessive-compulsive disorder: prevalence and clinical correlates. Depress Anxiety. 2012;29(11): 966-975

9. Jaisoorya TS, Reddy YC, Srinath S. The relationship of obsessivecompulsive disorder to putative spectrum disorders: results from an Indian study. Compr Psychiatry. 2003;44(4):317-323.

10. Jaisoorya TS, Janardhan Reddy YC, Srinath S. Is juvenile obsessivecompulsive disorder a developmental subtype of the disorder? Findings from an Indian study. Eur Child Adolesc Psychiatry. 2003;12(6): 290-297.

11. Lochner C, Fineberg NA, Zohar J, et al. Comorbidity in obsessivecompulsive disorder (OCD): a report from the International College of Obsessive-Compulsive Spectrum Disorders (ICOCS). Compr Psychiatry. 2014;55(7):1513-1519.

12. Lochner C, Stein DJ. Obsessive-compulsive spectrum disorders in obsessive-compulsive disorder and other anxiety disorders. Psychopathology. 2010;43(6):389-396.

13. Phillips KA, Gunderson CG, Mallya G, McElroy SL, Carter W. A comparison study of body dysmorphic disorder and obsessive-compulsive disorder. J Clin Psychiatry. 1998;59(11):568-575.

14. Simeon D, Hollander E, Stein DJ, Cohen L, Aronowitz B. Body dysmorphic disorder in the DSM-IV field trial for obsessive-compulsive disorder. Am J Psychiatry. 1995;152(8):1207-1209

15. Wilhelm S, Otto MW, Zucker BG, Pollack MH. Prevalence of body dysmorphic disorder in patients with anxiety disorders. J Anxiety Disord. 1997;11(5):499-502.

16. Brakoulias V, Starcevic V, Sammut P, et al. Obsessive-compulsive spectrum disorders: a comorbidity and family history perspective. Australas Psychiatry. 2011;19(2):151-155.

17. Brawman-Mintzer O, Lydiard RB, Phillips KA, et al. Body dysmorphic disorder in patients with anxiety disorders and major depression: a comorbidity study. Am J Psychiatry. 1995;152(11) 1665-1667.

18. Stewart SE, Stack DE, Wilhelm S. Severe obsessive-compulsive disorder with and without body dysmorphic disorder: clinical correlates and implications. Ann Clin Psychiatry. 2008;20(1):333-338.

19. Altamura C, Paluello MM, Mundo E, Medda S, Mannu P. Clinical and subclinical body dysmorphic disorder. Eur Arch Psychiatry Clin Neurosci. 2001;251(3):105-108.

20. Fontenelle LF, Telles LL, Nazar BP, et al. A sociodemographic, phenomenological, and long-term follow-up study of patients with body dysmorphic disorder in Brazil. Int J Psychiatry Med. 2006;36(2): 243-259.
21. Gunstad J, Phillips KA. Axis I comorbidity in body dysmorphic disorder. Compr Psychiatry. 2003;44(4):270-276.

22. Nierenberg AA, Phillips KA, Petersen TJ. Body dysmorphic disorder in outpatients with major depression. $J$ Affect Disord. 2002;69(1-3):141-148.

23. Phillips KA, Pagano ME, Menard W. Pharmacotherapy for body dysmorphic disorder: treatment received and illness severity. Ann Clin Psychiatry. 2006;18(4):251-257.

24. Hollander E, Allen A, Kwon J, et al. Clomipramine vs desipramine crossover trial in body dysmorphic disorder: selective efficacy of a serotonin reuptake inhibitor in imagined ugliness. Arch Gen Psychiatry. 1999;56(11):1033-1039.

25. van der Meer J, van Rood YR, van der Wee NJ, et al. Prevalence, demographic and clinical characteristics of body dysmorphic disorder among psychiatric outpatients with mood, anxiety or somatoform disorders. Nord J Psychiatry. 2012;66(4):232-238.

26. Zimmerman M, Mattia JI. Body dysmorphic disorder in psychiatric outpatients: recognition, prevalence, comorbidity, demographic, and clinical correlates. Compr Psychiatry. 1998;39(5):265-270.

27. Hollander E. Obsessive-compulsive spectrum disorders: an overview. Psychiatr Ann. 1993;23:355-358.

28. Monzani B, Rijsdijk F, Iervolino AC, Anson M, Cherkas L, MataixCols D. Evidence for a genetic overlap between body dysmorphic concerns and obsessive-compulsive symptoms in an adult female community twin sample. Am J Med Genet B Neuropsychiatr Genet. 2012;159B(4): 376-382.

29. Monzani B, Rijsdijk F, Harris J, Mataix-Cols D. The structure of genetic and environmental risk factors for dimensional representations of DSM-5 obsessive-compulsive spectrum disorders. JAMA Psychiatry. 2014;71(2):182-189.

30. Neziroglu F, Khemlani-Patel S, Yaryura-Tobias JA. Rates of abuse in body dysmorphic disorder and obsessive-compulsive disorder. Body Image. 2006;3(2):189-193.

31. Didie ER, Walters MM, Pinto A, et al. A comparison of quality of life and psychosocial functioning in obsessive-compulsive disorder and body dysmorphic disorder. Ann Clin Psychiatry. 2007;19(3): 181-186.

32. Frare F, Perugi G, Ruffolo G, Toni C. Obsessive-compulsive disorder and body dysmorphic disorder: a comparison of clinical features. Eur Psychiatry. 2004;19(5):292-298.

33. Phillips KA, Pinto A, Menard W, Eisen JL, Mancebo M, Rasmussen SA. Obsessive-compulsive disorder versus body dysmorphic disorder: a comparison study of two possibly related disorders. Depress Anxiety. 2007;24(6):399-409.

34. Tükel R, Tihan AK, Oztürk N. A comparison of comorbidity in body dysmorphic disorder and obsessive-compulsive disorder. Ann Clin Psychiatry. 2013;25(3):210-216.

35. Veale D, Ennis M, Lambrou C. Possible association of body dysmorphic disorder with an occupation or education in art and design. Am J Psychiatry. 2002;159(10):1788-1790.

36. Eisen JL, Phillips KA, Coles ME, Rasmussen SA. Insight in obsessive compulsive disorder and body dysmorphic disorder. Compr Psychiatry. 2004;45(1):10-15.

37. Phillips KA, Pinto A, Hart AS, et al. A comparison of insight in body dysmorphic disorder and obsessive-compulsive disorder. $J$ Psychiatr Res. 2012;46(10):1293-1299.

38. Reese HE, McNally RJ, Wilhelm S. Reality monitoring in patients with body dysmorphic disorder. Behav Ther. 2011;42(3):387-398.

39. McKay D, Neziroglu F, Yaryura-Tobias JA. Comparison of clinical characteristics in obsessive-compulsive disorder and body dysmorphic disorder. J Anxiety Disord. 1997;11(4):447-454.

40. Buhlmann U, Wilhelm S, McNally RJ, Tuschen-Caffier B, Baer L, Jenike MA. Interpretive biases for ambiguous information in body dysmorphic disorder. CNS Spectr. 2002;7(6):435-436.

41. Buhlmann U, McNally RJ, Etcoff NL, Tuschen-Caffier B, Wilhelm S. Emotion recognition deficits in body dysmorphic disorder. J Psychiatr Res. 2004;38(2):201-206. 
42. Reese HE, McNally RJ, Wilhelm S. Probabilistic reasoning in patients with body dysmorphic disorder. J Behav Ther Exp Psychiatry. 2011; 42(3):270-276.

43. Buhlmann U, Etcoff NL, Wilhelm S. Facial attractiveness ratings and perfectionism in body dysmorphic disorder and obsessive-compulsive disorder. J Anxiety Disord. 2008;22(3):540-547.

44. Reese HE, McNally RJ, Wilhelm S. Facial asymmetry detection in patients with body dysmorphic disorder. Behav Res Ther. 2010; 48(9):936-940.

45. Yaryura-Tobias JA, Neziroglu F, Chang R, Lee S, Pinto A, Donohue L. Computerized perceptual analysis of patients with body dysmorphic disorder: a pilot study. CNS Spectr. 2002;7(6):444-446.

46. Hart AS, Phillips KA. Symmetry concerns as a symptom of body dysmorphic disorder. J Obsessive Compuls Relat Disord. 2013;2(3): 292-298.

47. Diniz JB, Costa DL, Cassab RC, Pereira CA, Miguel EC, Shavitt RG. The impact of comorbid body dysmorphic disorder on the response to sequential pharmacological trials for obsessive-compulsive disorder. J Psychopharmacol. 2013;28(6):603-611.

48. Nakata AC, Diniz JB, Torres AR, et al. Level of insight and clinical features of obsessive-compulsive disorder with and without body dysmorphic disorder. CNS Spectr. 2007;12(4):295-303.

49. Phillips KA, Albertini RS, Rasmussen SA. A randomized placebocontrolled trial of fluoxetine in body dysmorphic disorder. Arch Gen Psychiatry. 2002;59(4):381-388.

50. Marazziti D, Giannotti D, Catena MC, et al. Insight in body dysmorphic disorder with and without comorbid obsessive-compulsive disorder. CNS Spectr. 2006;11(7):494-498.

51. Phillips KA, Pagano ME, Menard W, Fay C, Stout RL. Predictors of remission from body dysmorphic disorder: a prospective study. J Nerv Ment Dis. 2005;193(8):564-567.

52. Hanes KR. Neuropsychological performance in body dysmorphic disorder. J Int Neuropsychol Soc. 1998;4(2):167-171.

53. Marazziti D, Dell'Osso L, Presta S, et al. Platelet [3H]paroxetine binding in patients with OCD-related disorders. Psychiatry Res. 1999;89(3): $223-228$.
54. Saxena S, Winograd A, Dunkin JJ, et al. A retrospective review of clinical characteristics and treatment response in body dysmorphic disorder versus obsessive-compulsive disorder. J Clin Psychiatry. 2001;62(1): $67-72$.

55. Rossell SL, Harrison BJ, Castle DJ. Can understanding the neurobiology of body dysmorphic disorder (BDD) inform treatment? Australas Psychiatry. 2015;23(4):361-364.

56. Mullen R, Linscott RJ. A comparison of delusions and overvalued ideas. J Nerv Ment Dis. 2010;198(1):35-38.

57. Santín JM, Gálvez FM. Overvalued ideas: psychopathologic issues. Actas Esp Psiquiatr. 2011;39(1):70-74.

58. Buhlmann U, Reese HE, Renaud S, Wilhelm S. Clinical considerations for the treatment of body dysmorphic disorder with cognitive-behavioral therapy. Body Image. 2008;5(1):39-49.

59. Taillon A, O'Connor K, Dupuis G, Lavoie M. Inference-based therapy for body dysmorphic disorder. Clin Psychol Psychother. 2013; 20(1):67-76.

60. Moher D, Liberati A, Tetzlaff J, Altman DG; PRISMA Group. Preferred reporting items for systematic reviews and meta-analyses: the PRISMA statement. BMJ. 2009;339:b2535.

61. Veale D, Matsunaga H. Body dysmorphic disorder and olfactory reference disorder: proposals for ICD-11. Rev Bras Psiquiatr. 2014; 36(suppl 1):14-20.

62. Phillips KA, Wilhelm S, Koran LM, et al. Body dysmorphic disorder: some key issues for DSM-V. Depress Anxiety. 2010;27(6):573-591.

63. Kleinknecht RA, Dinnel DL, Kleinknecht EE, Hiruma N, Harada N. Cultural factors in social anxiety: a comparison of social phobia symptoms and Taijin kyofusho. J Anxiety Disord. 1997;11(2):157-177.

64. Schieber K, Kollei I, de Zwaan M, Martin A. Classification of body dysmorphic disorder - what is the advantage of the new DSM-5 criteria. J Psychosom Res. 2015;78(3):223-227.

65. Phillips KA, Stout RL. Associations in the longitudinal course of body dysmorphic disorder with major depression, obsessive-compulsive disorder, and social phobia. J Psychiatr Res. 2006;40(4):360-369.
Neuropsychiatric Disease and Treatment

\section{Publish your work in this journal}

Neuropsychiatric Disease and Treatment is an international, peerreviewed journal of clinical therapeutics and pharmacology focusing on concise rapid reporting of clinical or pre-clinical studies on a range of neuropsychiatric and neurological disorders. This journal is indexed on PubMed Central, the 'PsycINFO' database and CAS,

\section{Dovepress}

and is the official journal of The International Neuropsychiatric Association (INA). The manuscript management system is completely online and includes a very quick and fair peer-review system, which is all easy to use. Visit http://www.dovepress.com/testimonials.php to read real quotes from published authors. 